\title{
Norois
}

Environnement, aménagement, société

$240 \mid 2016$

Aménagement foncier, risque côtier, évolution des fleuves

\section{Étude comparative de l'évolution du trait de côte sur deux flèches sableuses de la Petite Côte (Sénégal) : cas de Joal et de Djiffère}

Comparative study of shoreline evolution on the sandspits of the "Petite Côte"

(Senegal): case of Joal and Diffère

Yves Birame Diadhiou, Abdoulaye Ndour, Isabelle Niang et Awa Niang-Fall

\section{(2) OpenEdition}

\section{Journals}

\section{Édition électronique}

URL : http://journals.openedition.org/norois/5935

DOI : $10.4000 /$ norois.5935

ISBN : 978-2-7535-5362-0

ISSN : $1760-8546$

\section{Éditeur}

Presses universitaires de Rennes

Édition imprimée

Date de publication : 30 novembre 2016

Pagination : 25-42

ISBN : 978-2-7535-5356-9

ISSN : 0029-182X

\section{Référence électronique}

Yves Birame Diadhiou, Abdoulaye Ndour, Isabelle Niang et Awa Niang-Fall, «Étude comparative de l'évolution du trait de côte sur deux flèches sableuses de la Petite Côte (Sénégal) : cas de Joal et de Djiffère », Norois [En ligne], 240 | 2016, mis en ligne le 30 novembre 2018, consulté le 02 janvier 2020 URL : http://journals.openedition.org/norois/5935; DOI : 10.4000/norois.5935 


Presses
Universitaires
de Rennes
www.pur-editions.fr

\title{
Étude comparative de l'évolution du trait de côte sur deux flèches sableuses de la Petite Côte (Sénégal) : cas de Joal et de Djiffère
}

\author{
Comparative Study of Shoreline Evolution on the Sandspits of the "Petite Côte" (Senegal): \\ Case of Joal and Djiffère
}

Yves Birame Diadhiou ${ }^{\mathrm{a} *}$, Abdoulaye Ndour $^{\mathrm{b}}$, Isabelle Niang $^{\mathrm{b}}$ et Awa Niang-FalL ${ }^{\mathrm{c}}$

\footnotetext{
*Auteur correspondant tel : (+221) 774292666

a Environnement Littoral, Faculté des Sciences et Techniques, Université Cheikh Anta Diop, Laboratoire de Sédimentologie, BP 5005 DAKAR-FAnN. (diadhiouyves@yahoo.fr)

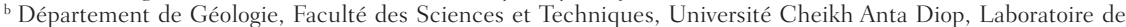
Sédimentologie, BP 5005 DAKAR-FAnN. (niangisabelle@yahoo.fr,abdoulayndour75@yahoo.fr)

c Département de Géographie, Faculté des Lettres et Sciences humaines Université Cheikh Anta Diop, BP 5005 DAKAR-FANN (n_awa@hotmail.com)
}

Résumé : Les flèches sableuses de Joal et de Djiffère, situées sur la Petite Côte du Sénégal, présentent des évolutions différentes. La cinématique du trait de côte est assez complexe à Joal contrairement à Palmarin-Djiffère où le taux de recul est graduel, en particulier aux extrémités nord et sud de la flèche. La tendance moyenne de recul des traits de côte digitalisés après correction géométrique (1989 à 2013) donne un taux de recul insignifiant de -0,82 m/an (20,4 m) à Joal et important à Palmarin-Djiffère (-3,83 m/an soit $91,92 \mathrm{~m}$ ). Les bilans sédimentaires trimestriels obtenus par suivi des profils de plage de janvier 2012 à septembre 2013 indiquent des échanges sédimentaires permettant d'identifier les mois de janvier, février, juillet et août comme des périodes d'érosion, et mars et septembre de reconstitution des plages. La dynamique de ces littoraux est en cohérence avec le régime des houles de la côte sud de Dakar et de la situation d'ouverture de la brèche de Sangomar.

\begin{abstract}
The sandy spits of Joal and Djiffere area, located on the Small Coast of Senegal, present different evolutions. The Joal shoreline's kinematics is complex in contrast with Palmarin-Djifferre where the rate of retreat is gradual especially at the north and south ends of the spit. The global average trend of shorelines retreat digitised after geometric correction (1989 to 2013) gives a retreat rate insignificant for Joal coast and important for Palmarin-Djiffère shoreline (respectively -0,82 m/year e.g.20,4 m and -3,83 m/year e.g. $91,92 \mathrm{~m}$ ). The quarterly sedimentary balances, obtained by beach profiles survey from January 2012 to September 2013, indicate sedimentary exchanges showing the months of january, february, july and august as periods of erosion, and march and september as mounths of beaches feeding. The dynamic of these coasts is consistent with the swells regime of the south coast of Dakar and with the Sangomar breach development.
\end{abstract}

Mots clés : littoral - trait de côte - flèche littorale - bilan sédimentaire

Keywords : coast-shoreline - sandspits - sediment budget 


\section{INTRODUCTION}

Dans le monde, les plages sableuses qui sont des formes d'accumulations sédimentaires évoluent à des vitesses variables (Bird, 1985, Paskoff, 1998). En Afrique de l'ouest, de la Mauritanie à la Sierra Léone les plages régressent à un taux estimé entre 1,2 et 6 m/an (PNUE, 1989; Cicin-Sain et Knecht, 1998; Faye et al., 2008; Faye, 2010). Les flèches sableuses à pointe libre sont aussi concernées par le processus. Au Sénégal, l'érosion est observée le long du littoral avec des variations spatio-temporelles des taux de recul, notamment sur la flèche de la Langue de Barbarie (Sall, 1982; Sy et Sy, 2010; Bâ, 2013, Sy et al., 2013), de Sangomar, de Mbodiène et de Joal (Sall, 1982; Diaw, 1997; Thomas et Diaw, 1997; Diara, 1999). La particularité de ces formes littorales est d'avoir généralement un ou des secteurs en érosion, la partie proximale et/ou la partie médiane en amont-dérive alimentant en aval-dérive une partie en progradation (partie distale) avec construction parfois de crochets. Ces deux phénomènes se produisent dans le sens de la dérive. Sur les flèches de Joal et de Djiffère, l'érosion est présente mais les manifestations morpho-sédimentaires et le rythme sont très contrastés entre les deux sites. De Joal à Palmarin, un recul global du trait de côte de 1,2 m/ an (Sall, 1982) et une avancée de $1 \mathrm{~m} / \mathrm{an}$ au sud de Joal de 1960 à 1989 (Niang-Diop, 1995) avaient été observés. La salinisation des terres et des nappes et l'inondation saisonnière (saison des pluies ou été) sont aussi observées. Les deux flèches littorales sableuses étudiées appartiennent au bassin sénégalo-mauritanien. Elles datent de la période postglaciaire (Post-nouakchottien) vers 4500 ans BP (Diaw, 1997). Leur évolution dépend largement du bilan sédimentaire entre les apports sédimentaires provenant en général de la dérive littorale (Diaw, 1997) et dans certains cas des apports éoliens ou fluviaux (Suanez, 2009) et les pertes occasionnées en partie par l'action des houles surtout de tempête et des activités humaines. Au Sénégal, le régime des houles dominant et la dérive induite d'une manière générale sont d'orientation NW. Des houles intermittentes de secteur SW avec une dérive de même direction (de juin à septembre) et de secteur W (entre octobre et décembre) sont observées. La houle de secteur NW et la dérive induite sont à l'origine du sens méridional pris par l'ensemble des flèches littorales
(Diaw, 1997) : Langue de Barbarie, Mbodiène, Joal, Sangomar etc. Les transports sédimentaires induits par la dérive varient en fonction des côtes. Ils sont de l'ordre de 100000 à $300000 \mathrm{~m}^{3}$ par an (Minot, 1934) sur la flèche de Sangomar et de l'ordre de $25000 \mathrm{~m}^{3}$ par an entre Rufisque et Joal. Les apports sédimentaires d'origine éolienne sont très faibles sur cette Petite Côte (Faye, 2010). En effet, sur cette partie du littoral, les vents ne dépassent pas $5 \mathrm{~m} / \mathrm{s}$. Les vitesses les plus fortes interviennent entre mars et août (tableau 1). Il s'agit des vents de secteur nord ou alizés maritimes pouvant atteindre $4,5 \mathrm{~m} / \mathrm{s}$ entre mars et avril ou des vents d'ouest ou mousson alternant avec des alizés maritimes de vitesses comprises entre 3 à $3,8 \mathrm{~m} / \mathrm{s}$ entre mai et août. Le marnage est faible et varie entre 1,2 à 1,6 m en marée de vive-eau et de 0,5 à 0,6 m en marée de morte-eau (Niang-Diop, 1995). Thiam (1986) avait trouvé une hauteur moyenne de 1,14 m en pleine mer, 0,55 m en basse mer et une amplitude moyenne de $0,58 \mathrm{~m}$ à Djiffère. Les courants qui en résultent participent à la dynamique côtière.

L'ouverture de la brèche de Sangomar vers Djiffère, provoquée par une tempête associée à une houle de forte amplitude (2,5 à 3,5 m) et de marées de vives eaux (Diaw, 1997) le 27 février 1987, a eu aussi un impact réel sur l'évolution de cette côte. Depuis, un recul de la pointe de Djiffère, des accumulations et des formations de crochons au sud sont notés (Diaw, 1997; Thomas et Diaw, 1997; Diara, 1999). La vitesse d'élargissement de la brèche est estimée à $214 \mathrm{~m} / \mathrm{an}$ entre 1987 et 2010 et à $145 \mathrm{~m} /$ an entre 1990 et 2010 (Dièye et al., 2013).

Sur cette côte de Joal à Djiffère, plusieurs activités humaines sont pratiquées : la pêche et les activités dérivées (transformation du poisson), le tourisme balnéaire et l'écotourisme, le ramassage de produits de mer, etc. Elles favorisent l'extension de la ville de Joal et le développement de campements touristiques le long de Palmarin-Djiffère et, parfois, le prélèvement de sédiments sur les plages pour la construction des maisons. L'extraction sectorielle du sable marin observée jadis, au nord de Joal et sur la pointe Sarène (Cesaraccio et al., 2004 ; Sy, 2007) a eu des influences sur l'évolution de la côte en réduisant la quantité de sédiments transportée par la dérive littorale.

Ainsi, plusieurs facteurs naturels et humains participent à la dynamique côtière. L'article présenté a 


\begin{tabular}{|c|c|c|c|c|c|c|c|c|c|c|c|c|}
\hline Années & Janv. & Févr. & Mars & Avr. & Mai & Juin & Juil. & Août & Sept & Oct. & Nov. & Déc. \\
\hline 2002 & 2,2 & 2,1 & 2,6 & 3,2 & 3,0 & 3,2 & 3,8 & 3,1 & 2,1 & 2,2 & 1,8 & 1,7 \\
\hline 2003 & 2,9 & 2,7 & 3,3 & 2,5 & 3,1 & 2,6 & 1,7 & 1,5 & 1,4 & 1,5 & 1,7 & 1,9 \\
\hline 2004 & 2,5 & 2,7 & 3,0 & 2,5 & 2,2 & 2,3 & 2,1 & 1,9 & 1,8 & 2,3 & 2,0 & 2,0 \\
\hline 2005 & 2,6 & 2,5 & 3,3 & 3,9 & 3,7 & 3,2 & 3,2 & 3,1 & 2,2 & 2,4 & 1,9 & 2,4 \\
\hline 2006 & 2,6 & 3,4 & 4,3 & 4,5 & 3,6 & 3,8 & 3,5 & 2,7 & 1,9 & 2,2 & 2,2 & 2,2 \\
\hline 2007 & 2,4 & 3,6 & 3,8 & 4,1 & 4,1 & 3,4 & 3,3 & 3,0 & 2,8 & 2,4 & 1,8 & 2,8 \\
\hline 2008 & 2,5 & 2,5 & 3,3 & 3,1 & 2,8 & 2,2 & 2,6 & 2,1 & 1,6 & 2,2 & 3,0 & 2,5 \\
\hline 2009 & 2,7 & 2,5 & 3,1 & 3,1 & 2,8 & 2,2 & 2,3 & 1,8 & 1,6 & 1,6 & 1,7 & 2,2 \\
\hline 2010 & 2,6 & 2,6 & 3,1 & 2,3 & 2,9 & 2,2 & 1,4 & 1,3 & 1,2 & 1,5 & 1,5 & 1,7 \\
\hline
\end{tabular}

Tableau 1 : Vitesse des vents en m/s à la station de Mbour entre 2002 et 2010 (Source : Agence Nationale de l'Aviation Civile et de la Météorologie du Sénégal, 2011)

Average annual monthly wind speed in m.s-1 in Mbour between 2002 to 2010

pour objectifs : i) de mesurer et comparer l'évolution du trait de côte sur la période qui suit l'ouverture de la brèche de Sangomar (1989 et 2013) à partir d'une photographie aérienne (1989), des images satellitaires (2002 et 2010) et des levés GPS (2013); et ii) de déterminer les échanges sédimentaires sur les plages aériennes des flèches littorales de Joal et de Djiffère de janvier 2012 à septembre 2013. Elle constitue un complément d'information des études antérieures réalisées sur cette zone côtière (Sall, 1982 ; Niang-Diop, 1995 ; Diaw, 1997 ; Diara, 1999; Turmine, 2000 et 2001 ; Sy, 2007 etc.). Elle a pour particularité de corréler cette évolution du trait de côte à la dynamique sédimentaire des plages aériennes, surtout dans le contexte des changements climatiques et de la gestion des risques.

\section{Présentation des flèches littorales de Joal et de Djiffère}

La flèche de « Djiffère » intégrée dans la plupart des études à la flèche de Sangomar et celle de Joal sont d'orientation respective NW-SE et NNWSSE (figure 1 - planche I). Elles se situent sur la Petite Côte sénégalaise, environ à $130 \mathrm{~km}$ au sud de Dakar et à $30 \mathrm{~km}$ de Mbour. Cette côte englobe la lagune de Joal-Fadiouth et une partie de l'estuaire du Saloum. La mangrove borde les marigots ou «bolons » parcourus par les ondes de marée et parfois en touffes isolées sur le cordon dunaire. La mangrove évolue soit au détriment des tannes soit en leur faveur. De façon générale, elle est régressive et en bon état uniquement le long des bolons (Dièye et al., 2013).

Les plages sableuses s'adossent par endroits à une microfalaise vive de hauteur variable mais inférieure à $2 \mathrm{~m}$ et entaillée localement dans des sables coquilliers. C'est le cas entre Palmarin Nguethie et Palmarin Ngallou. Dans les autres secteurs côtiers, les microfalaises sont moins marquées et ne s'observent qu'en périodes de forte agitation marine. À Joal, le tracé de la côte est irrégulier et le cordon est fortement anthropisé. Le cap Gaskel situé au nord du quai de pêche de Joal délimite une baie plus ou moins abritée mais exposée à la dynamique marine. À Palmarin- Djiffère, la côte est rectiligne. Les altitudes moyennes du cordon dunaire sont basses : $3 \mathrm{~m}$ en se basant sur les cartes topographiques de Palmarin-Djiffère ${ }^{1}$ et les levés altimétriques sur la flèche de Joal (figure 2 - planche II) réalisés au GPS différentiel. Ils ont servi à générer le modèle numérique de terrain et déterminer les pentes (figure 3 - planche II) sur la plage aérienne. Les pentes moyennes varient entre $0 \%$ sur le bas estran et $3 \%\left(1,72^{\circ}\right)$ sur le haut estran.

\section{MÉTHOdologie}

La photo-interprétation sous logiciel de traitement cartographique après correction géométrique ou redressement des données a été utilisée pour évaluer l'évolution du trait de côte. Pour déterminer les

1. feuilles Sokone 3C et 3D au 1/50000 de la mission JICA, 1989. 
échanges sédimentaires sur la plage aérienne (entre la haute plage et l'estran) de Joal et de Palmarin, le suivi trimestriel de profils de plage a permis de mesurer les échanges sédimentaires " cross-shore » sur des sections littorales de Joal et de PalmarinDjiffère, et ainsi quantifier un budget sédimentaire annuel.

\section{Évaluation de l'évolution du trait de côte}

\section{Données utilisées et choix des dates}

L'estimation de l'évolution du trait de côte a été faite en utilisant les données du tableau $2:$ i) deux scènes de photographie aérienne au 1/60000 de la mission JICA (Japon International Cooperation Agency) prises le 15 mars 1989 obtenues à la Direction des Travaux Géographique et Cartographique (DTGC); ii) une image Spot de résolution 10 m du 24 décembre 2002 acquise au Centre de Suivi Ecologique (CSE); iii) une capture d'image de Google Earth Pro d'avril 2010 et iv) des levés GPS (Garmin 12 XL 2D (XY) de précision 1 à 5 m de 2013 couvrant Joal et Palmarin. Rappelons que les données DGPS, citées ci-dessus, ne couvrent que Joal et ont été utilisées pour déterminer la variation des pentes sur l'estran. Les données acquises à la DTGC et au CSE ont été fournies en format numérique. L'image Spot a été corrigée et géoréférencée par le Centre de Suivi Écologique.

Les dates 1989 et 2002 ont été choisies car elles offrent une couverture globale de la zone et une bonne visibilité de l'interface terre-mer. 1989 est la seule image que nous avons pu obtenir et la plus proche de l'année de rupture de la flèche de Sangomar, tandis que 2002 a été choisie car elle est mentionnée par Ackermann et al. (2006) et Sy (2007) comme une période d'érosion à Joal. Les dates 2010 et 2013 ont été retenues puisqu'elles coïncident avec le début et la fin des missions des travaux de terrain (étude morphologique). Des contraintes d'acquisition des données pour ces deux dernières dates expliquent le recours à l'image extraite de Google Earth et des levés GPS 2D (XY).

\section{Correction géométrique des supports numériques}

La méthode classique de correction géométrique ou de rectification utilisée par plusieurs auteurs (Dolan et al., 1991; Durand et Heurtefeux, 2006; Faye et al., 2008; Bouabdallah et Larue, 2009) a été adoptée pour rendre les traits de côte comparables. Celle-ci a consisté à géo-référencer deux à deux les supports avec le logiciel Erdas en adoptant le mode polynomial d'ordre deux qui nécessite six amers pour lancer l'opération de correction. Nous avons utilisé sept amers bien répartis choisis sur l'image Spot déjà calée et identifiables sur les autres supports. Il s'agit des points d'intersection routiers et des points d'intersection de cours d'eau, seuls points remarquables communs repérables sur les supports et complétés par six points GPS de précision de 1 à $5 \mathrm{~m}$. La vérification de la parfaite superposition des routes et des cours d'eau des supports s'est faite avec l'outil «swipe ». Après correction, le trait de côte choisi (la ligne supérieure d'humectation des sables) de chaque support a été digitalisé dans ArcGIS 9.3. Cette ligne de référence qui matérialise le niveau atteint par les pleines mers sur l'estran était plus facile à détecter sur la photo aérienne et l'image Spot que le pied de dune et la ligne de rivage instantanée. Elle est aussi le plus fréquemment utilisée dans les études menées sur cette zone (Sy, 2007 ; Deec, 2010). Les résultats peuvent être d'une précision de l'ordre métrique, même si Faye et al., (2008) soulignent l'incertitude de plus en plus grande si les supports sont de mauvaises qualités, en plus de l'effet de la marée changeante à prendre en compte.

\begin{tabular}{|c|c|c|c|}
\hline Années & Échelle des clichés & Organisme & Taille du Pixel (m) \\
\hline 1989 & $1 / 60000$ & Mission JICA et DTGC & 5,00 \\
\hline 2002 & $10 \mathrm{~m}$ & Spot acheté par le CSE & 5,5 \\
\hline 2010 & $?$ & Site Google Earth & 8,29 \\
\hline
\end{tabular}

Tableau 2 : Données utilisées pour déterminer l'évolution du trait de côte à Joal et Palmarin-Djiffère Data used to determinate shoreline evolution on Joal and Palmarin-Djiffère spits 


\section{Détermination des erreurs}

Les erreurs liées à la méthode adoptée sont multiples (Dolan et al, 1991; Durand et Heurtefeux, 2006; Faye et al., 2008; Bouabdallah et Larue, 2009). Il s'agit généralement des erreurs de calage, des erreurs induites par la marée et des erreurs liées à la qualité des supports. Les erreurs liées à la qualité des supports (la visibilité du trait de côte) sont difficiles à déterminer et nécessitent l'obtention de plusieurs informations sur les supports (Faye, 2010).

Les erreurs de superposition ou de calage sont données par le logiciel en Root Mean Square (RMS). Les RMS obtenus sont de 0,6 pixel $(6 \mathrm{~m})$ entre l'image Spot et les photos aériennes et de 0,5 pixel $(5 \mathrm{~m})$ entre l'image Spot et l'image extraite de Google Earth. Cette erreur a été ramenée à $10 \mathrm{~m}$ c'est-à-dire à deux fois la taille du pixel qui est de $5 \mathrm{~m}$ et qui correspond aussi à la résolution de l’image de référence.

L'erreur due à la marée a été calculée approximativement en déterminant la différence entre les niveaux moyens des pleines mers et celui des basses mers. La formule de Thomas et Diaw, (1997) reprise par Faye et al. (2008) : $\Delta \mathrm{d}(\mathrm{m})=\mathrm{h} / \tan \theta(\Delta \mathrm{d}$ est l'erreur liée à la marée, $\theta$ la pente et h est la hauteur d'eau) a été utilisée.

Cette partie du littoral n'ayant pas de marégraphe, nous avons considéré les données moyennes des pleines mers de vives-eaux $(1,14 \mathrm{~m})$ et des basses marées de mortes eaux (0,55 m) mesurée entre 1985 et 1986 par Thiam (1986) pour estimer approximativement les erreurs liées à l'effet de la marée. La différence entre les deux hauteurs de marée est de 0,59 m. Les pentes mesurées sur le terrain sont de $0 \%$ sur le bas estran et de $3 \%\left(1,72^{\circ}\right)$ sur le haut estran. L'application de la formule donne pour une pente de $0^{\circ}$ une valeur nulle et pour une pente de $1,72^{\circ}$ une valeur de $19,66 \mathrm{~m}$ soit en moyenne $\pm 9,83 \mathrm{~m}(10 \mathrm{~m})$. D'autre part, la côte étant classée dans la catégorie des côtes microtidales, le marnage est faible et les écarts entre les pleines mers ne sont pas grands.

La précision du GPS utilisée variant entre \pm 1 et $5 \mathrm{~m}$ selon la notice du constructeur, nous avons considéré la valeur de $5 \mathrm{~m}$ comme erreur. Le cumul total des erreurs revient à $+/-25 \mathrm{~m}$. La marge d'erreur pour une période donnée a été à chaque fois requise par le « Digital shoreline analysis system » (Dsas 4.2) pour le calcul des taux d'évolution.

\section{Calcul des taux d'évolution}

Le calcul des taux d'évolution est fait automatiquement avec «Digital shoreline analysis system » (Dsas 4.2) implanté dans ArcGIS 9.3 en fixant sur la boîte de dialogue un espacement de 50 m entre les transects et en intégrant à chaque fois les marges d'erreur. Les résultats sont générés par le Dsas sous forme de tableaux attributaires avec l'indice End Point Rate (EPR) en taux. L'EPR traduit l'évolution calculée entre la distance de deux points situés sur deux lignes de rivage en fonction des dates de prise de vue (jours, mois, années). Les résultats ont été exportés dans Excel où les calculs du taux d'évolution global du trait de côte et des taux moyens sur les parties proximales, médianes et distales des flèches ont été faits.

\section{Suivi morpho-sédimentaire}

Le suivi trimestriel de profils topographiques de plages a été réalisé en saison sèche (janvier, février et mars) et pluviale (juillet, août et septembre) de 2012 à 2013. Ce choix est basé sur les connaissances empiriques préalables, collectées auprès des populations locales mentionnant décembre, janvier, février, juin, juillet et août comme périodes d'érosion. Nous avons soustrait arbitrairement décembre et juin. À Palmarin-Djiffère, ce suivi est limité à la partie médiane et nord de la flèche. Il concerne 7 profils de plage à Joal et 9 à Palmarin (figure 4 - planche III). Ces profils ont été réalisés avec un niveau optique et une mire de chantier à partir de bornes fixes. L'outil «Profiler » (complément sur Excel) a permis de tracer, de superposer les profils de plage deux à deux (figure 5) et de calculer les volumes sédimentaires en mètre cube par mètre linéaire entre 2012 et 2013 sur chaque profil. Les erreurs (de centrage à la borne, de lecture, de changement de borne, etc.), peuvent être négligées dans la mesure où les variations morphosédimentaires sont relativement faibles à un mètre de part et d'autre des profils. Les unités de la plage aérienne ont été subdivisées en identifiant la limite de la dernière laisse de haute mer et le niveau de la limite inférieure de l'estran. Les largeurs de la 


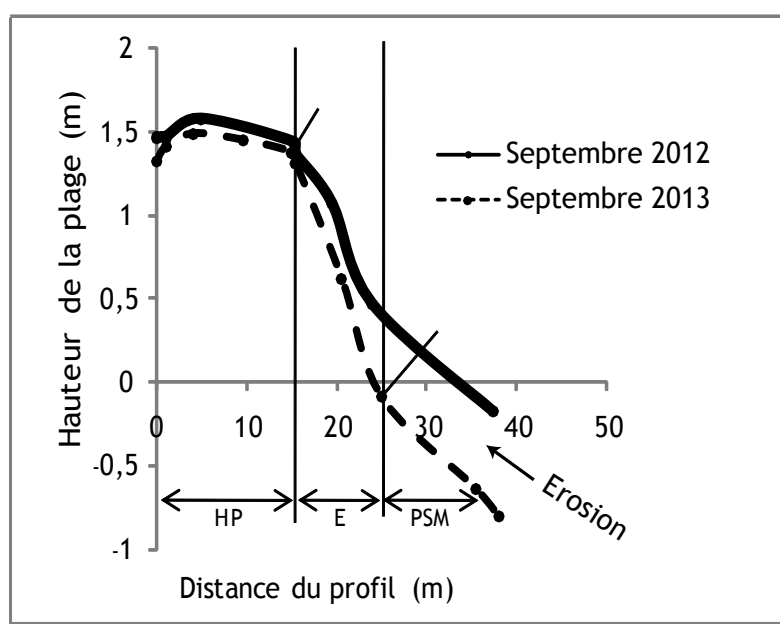

PJ16 (quai de pêche) sur la flèche de JOAL

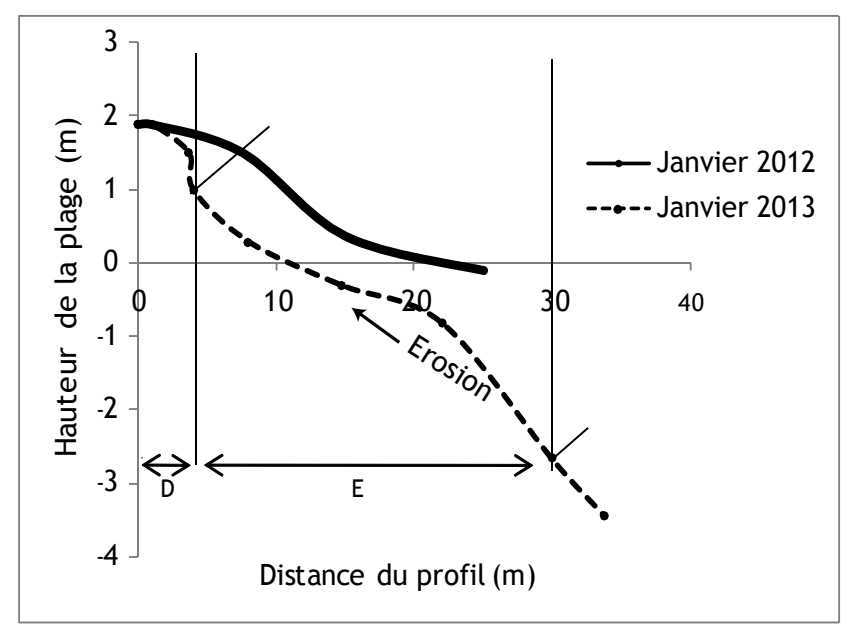

P1 (Diakhanor) sur Palmarin-Djiffère

Figure 5 : Comparaison de deux profils réalisés en janvier 2012 et 2013 à Joal (PJ6) et à Palmarin (P1) Comparison of two profiles realised in January 2012 and 2013 in Joal (PJ6) and in Palmarin (P1)

plage sous marine mesurées ont été très faibles ( 1 à $3 \mathrm{~m}$ ) ou pas souvent exploitée. Ce qui fait que seul le bilan sédimentaire sur la plage aérienne (haute plage + estran) a été pris en compte dans l'analyse des résultats.

Les profils PJ5 et PJ7 sur la flèche de Joal n'ont pas été suivis durant toute la période d'étude à cause des pertes des repères dues à l'activité anthropique et à l'érosion marine, de même que le profil P9 perdu à cause de l'accentuation de l'érosion

\section{RÉSultats}

\section{Évolution pluriannuelle et interannuelle du trait de côte}

Les résultats obtenus (tableau 3) montrent qu'à Joal, dans beaucoup de secteurs, les évolutions sont négligeables car elles sont inférieures à la marge d'erreur. Cependant, des évolutions considérables sont observées sur certains secteurs en fonction des périodes considérées. Entre 1989 et 2002, une érosion de 2,49 m/an est notée sur la partie nord et entre 1989 et 2010, une érosion de 1,56 m/an sur la partie sud. Les accumulations sont intervenues qu'entre 2002 et 2010 sur la partie nord $(5,28 \mathrm{~m} / \mathrm{an})$ et médiane (3,25 m/an) et entre 2002 et 2013 sur la partie nord (2,5 m/an). La flèche s'est allongée au cours de ces périodes.

Sur le secteur de Palmarin-Djiffère, situé au nord de la brèche de Sangomar et au sud de la flèche de
Joal, l'érosion est généralisée. Les évolutions insignifiantes sont notées qu'entre 1989 et 2002 sur les parties nord et médiane, et entre 2002 et 2010 sur la partie nord. Les parties nord et sud comprises respectivement entre Palmarin-Nguéthie et PalmarinNgallou et entre Palmarin Diakhanor et Djiffère (figures 6b et 6c - planche IV) reculent le plus.

Entre 1989 et 2010, le taux global de recul du trait de côte est de 2,61 m/an soit 54,81 m. Ce taux est de $-3,83 \mathrm{~m} / \mathrm{an}$ pour la période $1989-2013$, soit un recul de $91,92 \mathrm{~m}$.

La pointe de Djiffère recule mais la vitesse de régression est relativement faible entre 2002-2013 (2,4 m/an) comparée à 1989-2002 (1 629,94 m soit $-25,38 \mathrm{~m} / \mathrm{an})$.

\section{Échanges sédimentaires trimestriels et variation du trait de côte}

Le suivi topographique trimestriel des profils de plage montre des variations côtières importantes, même si les observations sont faites sur une période très courte pour donner une situation exhaustive du fonctionnement saisonnier des plages. Les mouvements sédimentaires " cross-shore " (transversaux) s'accompagnent d'échanges sédimentaires qui modifient constamment la position du trait de côte. Ainsi, le constat global est la prédominance de l'érosion. Les plages aériennes subissent des pertes sédimentaires (tableau 4 et 5) en particulier 


\begin{tabular}{|c|c|c|c|c|c|c|}
\hline \multirow[b]{3}{*}{ Secteurs } & \multicolumn{6}{|c|}{ Évolution du trait de côte (m/an) } \\
\hline & \multicolumn{6}{|c|}{ Flèche de Joal } \\
\hline & $\begin{array}{c}1989-2002 \\
(13 \text { ans })\end{array}$ & $\begin{array}{l}2002-2010 \\
\quad(8 \text { ans })\end{array}$ & $\begin{array}{c}2010-2013 \\
(3 \text { ans })\end{array}$ & $\begin{array}{c}2002-2013 \\
(11 \text { ans })\end{array}$ & $\begin{array}{c}1989-2010 \\
(21 \text { ans })\end{array}$ & $\begin{array}{c}1989-2013 \\
(24 \text { ans })\end{array}$ \\
\hline Nord & $-2,49$ & $+5,28$ & 1 & $+2,5$ & l & 1 \\
\hline Médiane & I & $+3,27$ & l & I & l & l \\
\hline Sud & I & I & I & $-3,04$ & $-1,56$ & I \\
\hline Moyenne annuelle & I & I & l & I & l & l \\
\hline \multirow[t]{2}{*}{ Allongement de la flèche } & $+20,4$ & I & $+11,7$ & $+8,07$ & $+14,63$ & $+1,77$ \\
\hline & \multicolumn{6}{|c|}{ Flèche de Djiffère } \\
\hline Nord & I & I & $-13,26$ & $-7,31$ & $-1,98$ & $-3,92$ \\
\hline Médiane & I & $-3,65$ & $-11,15$ & $-6,34$ & $-0,64$ & $-2,03$ \\
\hline Sud & $-4,56$ & $-7,07$ & $-11,09$ & $-7,88$ & $-5,23$ & $-5,65$ \\
\hline Moyenne annuelle & / & $-4,45(35,6 \mathrm{~m})$ & $-11,63(34,89)$ & $\begin{array}{c}-7,15 \\
(79,75 \mathrm{~m})\end{array}$ & $\begin{array}{c}-2,61 \\
(54,81 \mathrm{~m})\end{array}$ & $\begin{array}{c}-3,83 \\
(91,92 \mathrm{~m})\end{array}$ \\
\hline Recul de la pointe & $-125,38$ & $-5,84$ & 1 & $-2,4$ & $-85,72$ & $-60,6$ \\
\hline Marges d'erreur (m/an) & $\pm 1,9 \mathrm{~m}$ & $\pm 3,12$ & $\pm 8,3$ & $\pm 2,3$ & $\pm 1,19$ & $\pm 1,04$ \\
\hline
\end{tabular}

Tableau 3 : Taux d'évolution du trait de côte à Joal et Palmarin-Djiffère par période d'étude entre 1989 et 2013 (m/an). (/) taux non-significatifs car inférieurs aux marges d'erreur

Shoreline evolution rate in Joal and Palmarin-Djiffère spits between 1989 and 2013 (m/year). (/) Nonsignificant rate because of higher margin of error

les hautes plages se rétrécissent avec formation de microfalaises sur certaines parties (figures $7 \mathrm{a}, 7 \mathrm{~b}, 7 \mathrm{c}$ et $7 \mathrm{~d})$. Les mois de janvier et février puis de juillet et août sont des périodes d'érosion intense. Mars et septembre sont des périodes de reconstitution partielle des profils de plage. Les échanges sédimentaires défavorables à la haute plage sont plus accentués à Palmarin-Djiffère qu'à Joal où la plage aérienne subit des périodes d'accrétion. Le trait de côte suit généralement la même tendance : un recul du trait de côte qui se traduit par des érosions et une avancée qui s'accompagnent d'accrétion. Ces déficits qui s'accompagnent de modifications des plages concourent, à long terme, à des reculs du trait de côte et au rétrécissement progressif du cordon dunaire noté sur la flèche de Djiffère. À Joal, une forte variabilité spatiale caractérise le bilan sédimentaire trimestriel avec des excédents par moments surtout sur la partie nord : PJ4 et PJ6, et sud : PJ1 (tableau 5).

\section{Discussion}

L'étude de la cinématique du trait de côte et l'évaluation du fonctionnement des plages exigent de travailler sur des séries de données et des intervalles de temps longues et aussi d'adopter une méthode minimisant les erreurs. Les marges d'erreur importantes sont en partie liées aux cumuls des erreurs sur les supports, en particulier l'erreur induite par la marée qui a été obtenue approximativement et a rendu insignifiants certains taux trouvés surtout à Joal. Dans la plupart des études réalisées à Joal (Sall, 1982; Sy, 2007 ; Deec, 2010), à l'exception de Faye (2010) pour l'évaluation de l'évolution de la pointe de la flèche de Joal, l'erreur induite par la marée n'est pas prise en compte ou indiquée. Ainsi, les données trouvées sont comparables. Les séquences temporelles considérées dans cette étude et les résultats obtenus, corrélés aux données de la littérature (Sall, 1982 ; Niang-Diop 1995; Diaw, 1997; Thomas et Diaw, 1997; Diara, 1999; Turmine 2000 et 2001 ; Sy, 2007; Dièye et al., 2013) permettent d'analyser les variations globales et récentes sur les deux sites 


\begin{tabular}{|c|c|c|c|c|}
\hline \multicolumn{5}{|c|}{ Janvier 2012-2013 } \\
\hline Profils & $\mathrm{HP}$ & $\mathrm{E}$ & PA & PSM \\
\hline $\mathrm{Pl}$ & $-0,036$ & $-1,071$ & $-1,101$ & - \\
\hline $\mathrm{P} 2$ & $+0,049$ & $+1,773$ & $+1,822$ & - \\
\hline P3 & $-0,017$ & $+0,382$ & $-0,399$ & - \\
\hline $\mathrm{P} 4$ & $-0,105$ & $-0,100$ & $-0,205$ & $-0,339$ \\
\hline P5 & $-0,567$ & $-0,890$ & $-1,457$ & $-1,986$ \\
\hline P6 & $-0,146$ & $-1,624$ & $-1,270$ & - \\
\hline P7 & $-0,010$ & $-0,293$ & $-0,303$ & $-0,048$ \\
\hline P8 & $-0,079$ & $-0,002$ & $-0,081$ & $-1,170$ \\
\hline P9 & $-0,719$ & $-0,895$ & $-1,614$ & - \\
\hline \multicolumn{5}{|c|}{ Février 2012-2013 } \\
\hline Profils & $\mathrm{HP}$ & $\mathrm{E}$ & PA & PSM \\
\hline P1 & $-0,282$ & $+0,023$ & $-0,129$ & - \\
\hline $\mathrm{P} 2$ & $-0,137$ & $+0,075$ & $-0,106$ & $+0,147$ \\
\hline P3 & $+0,009$ & $+0,192$ & $+0,1$ & - \\
\hline P4 & $-0,293$ & $-0,645$ & $-0,469$ & - \\
\hline P5 & $-0,060$ & $-0,139$ & $-0,099$ & $+1,976$ \\
\hline P6 & $+0,018$ & $-1,560$ & $-0,771$ & - \\
\hline P7 & $-0,053$ & $-1,322$ & $-0,687$ & - \\
\hline P8 & $-0,170$ & $+0,111$ & $-0,029$ & - \\
\hline P9 & $-0,463$ & $-1,078$ & $-0,77$ & $-0,805$ \\
\hline \multicolumn{5}{|c|}{ Mars 2012-2013 } \\
\hline Profils & $\mathrm{HP}$ & $E$ & PA & PSM \\
\hline $\mathrm{Pl}$ & $-0,251$ & $-1,069$ & $-0,66$ & - \\
\hline P2 & $-0,179$ & $-1,475$ & $-0,827$ & - \\
\hline P3 & $-0,034$ & $-0,065$ & $-0,049$ & - \\
\hline $\mathrm{P} 4$ & $-0,029$ & $-1,173$ & $-0,061$ & - \\
\hline P5 & $+0,027$ & $-0,484$ & $-0,228$ & - \\
\hline P6 & $-0,139$ & $-0,265$ & $-0,202$ & $-0,87$ \\
\hline P7 & $-0,087$ & $-0,382$ & $-0,234$ & $-0,448$ \\
\hline P8 & $-0,251$ & -0.393 & $-0,322$ & $-1,517$ \\
\hline P9 & $-1,319$ & $-1,881$ & $-1,6$ & - \\
\hline
\end{tabular}

\begin{tabular}{|c|c|c|c|c|}
\hline \multicolumn{5}{|c|}{ Juillet 2012-2013 } \\
\hline Profils & HP & $\mathrm{E}$ & $\mathrm{PA}$ & PSM \\
\hline $\mathrm{Pl}$ & $-0,189$ & $+0,327$ & $+0,069$ & $-0,373$ \\
\hline P2 & $-0,039$ & $+0,453$ & $+0,207$ & $+1,507$ \\
\hline P3 & $-0,019$ & $+0,238$ & $+0,109$ & $+1,555$ \\
\hline $\mathrm{P} 4$ & $-0,180$ & $+0,054$ & $-0,063$ & $+0,967$ \\
\hline P5 & $-0,100$ & $-0,362$ & $-0,231$ & $+0,436$ \\
\hline P6 & $-0,225$ & $+0,063$ & $-0,081$ & $+1,361$ \\
\hline P7 & $+0,063$ & $+0,095$ & $+0,079$ & $+0,638$ \\
\hline P8 & $-0,197$ & $+0,212$ & $+0,007$ & $+0,259$ \\
\hline P9 & $-0,662$ & $-0,063$ & $-0,362$ & - \\
\hline \multicolumn{5}{|c|}{ Août 2012-2013 } \\
\hline Profils & HP & E & PA & PSM \\
\hline $\mathrm{Pl}$ & $-0,189$ & $+0,315$ & $+0,063$ & - \\
\hline $\mathrm{P} 2$ & $-0,039$ & $+0,430$ & $+0,195$ & $+1,504$ \\
\hline P3 & $-0,019$ & $+0,238$ & $+0,109$ & $+1,555$ \\
\hline $\mathrm{P} 4$ & $-0,180$ & $+0,054$ & $-0,063$ & $+0,967$ \\
\hline P5 & $-0,155$ & $-0,388$ & $-0,271$ & $+0,323$ \\
\hline P6 & $-0,225$ & $-0,102$ & $-0,163$ & $+1,188$ \\
\hline P7 & $+0,063$ & $+0,095$ & $+0,079$ & $+0,638$ \\
\hline P8 & $-0,197$ & $+0,212$ & $+0,007$ & $+0,259$ \\
\hline \multicolumn{5}{|c|}{ Septembre 2012-2013 } \\
\hline Profils & HP & $\mathrm{E}$ & $\mathrm{PA}$ & PSM \\
\hline $\mathrm{Pl}$ & $-0,017$ & $-0,414$ & $-0,584$ & - \\
\hline P2 & $+0,073$ & $+0,509$ & $+0,592$ & - \\
\hline P3 & $-0,224$ & $-0,402$ & $-0,622$ & - \\
\hline $\mathrm{P} 4$ & $-0,043$ & $+0,074$ & $+0,031$ & $+0,133$ \\
\hline P5 & $-0,291$ & $-0,459$ & $-0,750$ & $-0,184$ \\
\hline P6 & $-0,461$ & $-0,482$ & $-0,973$ & $-0,026$ \\
\hline P7 & $+0,089$ & $-0,340$ & $-0,251$ & $-0,210$ \\
\hline P8 & $-0,088$ & $-0,256$ & $-0,344$ & $-0,069$ \\
\hline
\end{tabular}

Tableau 4 : Bilan sédimentaire trimestriel en $\mathrm{m}^{3}$ par m linéaire entre Diakanor et Ngallou, Haute plage (HP), Estran (E), Plage Aérienne $(\mathrm{PA}=\mathrm{HP}+\mathrm{E})$ et Plage Sous-Marine (PSM)

Quarterly sedimentary balance in $\mathrm{m}^{3} \mathrm{~m}^{-1}$ linear between Diakhanor and Ngallou

étudiés et la dynamique sur d'autres sites. Ainsi, les changements notés sont assez cohérents par rapport à la tendance décrite par les auteurs cités ci-dessus et aux processus observés sur les autres flèches littorales sénégalaises (Langue de Barbarie, Mbodiène), sur d'autres littoraux sableux d'Afrique de l'Ouest, notamment le littoral de la Mauritanie ou du Maroc (Faye et al., 2008; Bouabdallah et Larue, 2009). Le caractère aléatoire de l'évolution du trait de côte à Joal mentionné par Niang-Diop (1995), Turmine
(2000 et 2001) se manifeste. Ces fluctuations dans l'évolution du trait de côte entraînent des phénomènes de compensation qui expliqueraient la classification de ce littoral dans la catégorie des côtes stable (Faye, 2010), et qui se traduisent dans cette étude par des taux faibles qui tombent dans la marge d'erreur comparés à la flèche de Palmarin-Djiffère. En effet, entre 2002 et 2010 et entre 2002 et 2013, la progradation respective de $+5,28 \mathrm{~m} / \mathrm{an}$ et de $+2,5 \mathrm{~m} / \mathrm{an}$ observée sur la plage du quai de pêche de 
Yves BIRAMe DiadHIOU - Norols nº $240(2016 / 3)$ p. 25-42

\begin{tabular}{|c|c|c|c|c|}
\hline \multicolumn{5}{|c|}{ Janvier 2012-2013 } \\
\hline Profils & $\mathrm{HP}$ & $\mathrm{E}$ & PA & PSM \\
\hline PJl & $-0,017$ & $+0,266$ & $+0,249$ & $+0,309$ \\
\hline $\mathrm{PJ} 2$ & $-0,389$ & $-0,340$ & $-0,729$ & - \\
\hline $\mathrm{PJ} 3$ & $-0,029$ & $+1,089$ & $+1,060$ & $+3,393$ \\
\hline PJ4 & $-0,040$ & $-0,409$ & $-0,449$ & - \\
\hline PJ5 & $-0,217$ & $-0,133$ & $-0,350$ & - \\
\hline PJ6 & $-0,272$ & $-0,125$ & $-0,397$ & - \\
\hline PJ7 & $-0,004$ & $-0,045$ & $-0,049$ & - \\
\hline \multicolumn{5}{|c|}{ Févier 2012-2013 } \\
\hline Profils & HP & $\mathrm{E}$ & $\mathrm{PA}$ & PSM \\
\hline PJl & 0,431 & 1,646 & $+1,038$ & - \\
\hline $\mathrm{PJ} 2$ & $-0,436$ & $-0,486$ & $-0,461$ & $-0,277$ \\
\hline PJ3 & $-0,137$ & $+0,034$ & $-0,051$ & $+0,059$ \\
\hline PJ4 & $-0,848$ & $-0,328$ & $-0,588$ & - \\
\hline PJ5 & $-0,058$ & $-0,117$ & $-0,087$ & $-0,128$ \\
\hline PJ6 & $-0,123$ & $-0,616$ & $-0,369$ & - \\
\hline PJ7 & $-0,001$ & $-0,175$ & $-0,088$ & $-0,212$ \\
\hline \multicolumn{5}{|c|}{ Mars 2012-2013 } \\
\hline Profils & $\mathrm{HP}$ & E & PA & PSM \\
\hline PJl & $+0,230$ & $+1,007$ & $+0,618$ & - \\
\hline $\mathrm{PJ} 2$ & $-0,400$ & $-0,566$ & $-0,483$ & $-0,498$ \\
\hline $\mathrm{PJ} 3$ & $-0,084$ & $-0,187$ & $-0,135$ & $-0,190$ \\
\hline PJ4 & $+0,203$ & $+0,635$ & $+0,419$ & - \\
\hline PJ5 & $-0,113$ & 0,189 & $+0,038$ & - \\
\hline PJ6 & $-0,207$ & $-0,489$ & $-0,348$ & - \\
\hline
\end{tabular}

\begin{tabular}{|c|c|c|c|c|}
\hline \multicolumn{5}{|l|}{ Juillet 2012-2013 } \\
\hline Profils & HP & E & PA & PSM \\
\hline PJ1 & $+0,006$ & $+0,997$ & $+0,501$ & $/$ \\
\hline PJ2 & $-0,663$ & $-0,884$ & $-0,773$ & $/$ \\
\hline PJ3 & $-0,073$ & $-0,167$ & $-0,12$ & $/$ \\
\hline PJ4 & $+0,251$ & $+0,490$ & $+0,37$ & $+0,458$ \\
\hline PJ6 & $-0,031$ & $-0,488$ & $-0,259$ & $/$ \\
\hline Aont $2012-2013$ & \multicolumn{5}{|l}{} \\
\end{tabular}

Août 2012-2013

\begin{tabular}{|c|c|c|c|c|}
\hline Profils & HP & E & PA & PSM \\
\hline PJ1 & $+0,139$ & $+0,930$ & $+0,534$ & $/$ \\
\hline PJ2 & $-0,282$ & $-0,716$ & $-0,449$ & $/$ \\
\hline PJ3 & $-0,267$ & $-0,654$ & $-0,460$ & $-0,522$ \\
\hline PJ4 & $+0,062$ & $+0,382$ & $+0,222$ & $+0,177$ \\
\hline PJ6 & $+0,030$ & $-0,337$ & $-0,153$ & $/$ \\
\hline
\end{tabular}

Septembre 2012-2013

\begin{tabular}{|c|c|c|c|c|}
\hline Profils & HP & E & PA & PSM \\
\hline PJ1 & $+0,506$ & $/$ & $/$ & $/$ \\
\hline PJ2 & $-0,237$ & $-0,321$ & $-0,558$ & $/$ \\
\hline PJ3 & $-0,209$ & $-0,512$ & $-0,721$ & $-0,599$ \\
\hline PJ4 & $+0,152$ & $+0,635$ & $+0,787$ & $+0,222$ \\
\hline PJ6 & $-0,100$ & $-0,385$ & $-0,485$ & $-0,536$ \\
\hline
\end{tabular}

Tableau 5 : Bilan sédimentaire trimestriel en $\mathrm{m}^{3}$ par $\mathrm{m}$ linéaire à Joal, Haute plage (HP), Estran (E), Plage Aérienne (PA = HP + E) et Plage Sous-Marine (PSM)

Quarterly sedimentary balance in $\mathrm{m}^{3} \mathrm{~m}^{-1}$ linear in Joal

Joal contraste avec l'érosion notée sur cette partie de la côte par Sy (2007) entre 1997 et $2002(-1,47$ m/ an). Cette fluctuation du trait de côte traduit une dynamique marquée tantôt par des accrétions, tantôt par des érosions (figure 8).

À Palmarin-Djiffère, les taux de recul de $7 \mathrm{~m} /$ an observés entre 2002 et 2013 concordent avec les tendances actuelles mentionnées par la Deec (2010). Le recul de la pointe de Djiffère se poursuit depuis l'ouverture de la brèche dont font état plusieurs auteurs (Diaw, 1997; Thomas et Diaw, 1997 ; Dièye et al., 2013). Diagne (2015) indique que, depuis son ouverture, la partie allant de PalmarinDiakhanor à Djiffère est devenue une zone de départ des sédiments et plus au sud une zone d'accumulation. Les évolutions des parties proximale, médiane et distale de la flèche de Joal et PalmarinDjiffère sont similaires à celles notées sur la flèche de Mbodiène et la Langue de Barbarie qui sont soumises à des périodes de recul et d'avancée du trait

de côte; et qui présentent des secteurs en érosion et des secteurs en accumulation. Entre 1954 et 2002, les parties proximales de la flèche Mbodiène et Joal ont connu respectivement une progression et une stabilité. Les parties médianes ont subi successivement des érosions de 1,6 m/an à Mbodiène (NgamiNtsiba- Andzou, 2006) et de 1,46 m/an à Joal (Sy, 2007). Les extrémités sont caractérisées par une très grande mobilité. Entre 1954 et 1989, la Langue de Barbarie a connu la même évolution : une érosion faible de $0,5 \mathrm{~m} / \mathrm{an}$ comparé au recul de 1 à $2 \mathrm{~m} / \mathrm{an}$ noté sur cette côte entre 1856 et 1926 avec déplacement du processus d'une extrémité à l'autre (NiangDiop, 1995).

En l'absence d'aménagements côtiers notables (épis, brise-lames, murs de protection) à part les emprises des établissements touristiques, l'évolution est liée à des causes naturelles. L'analyse croisée entre dynamique sédimentaire et du trait de côte par rapport aux manifestations des houles (Nardari, 1993), la morphologie des deux flèches, la vitesse et 


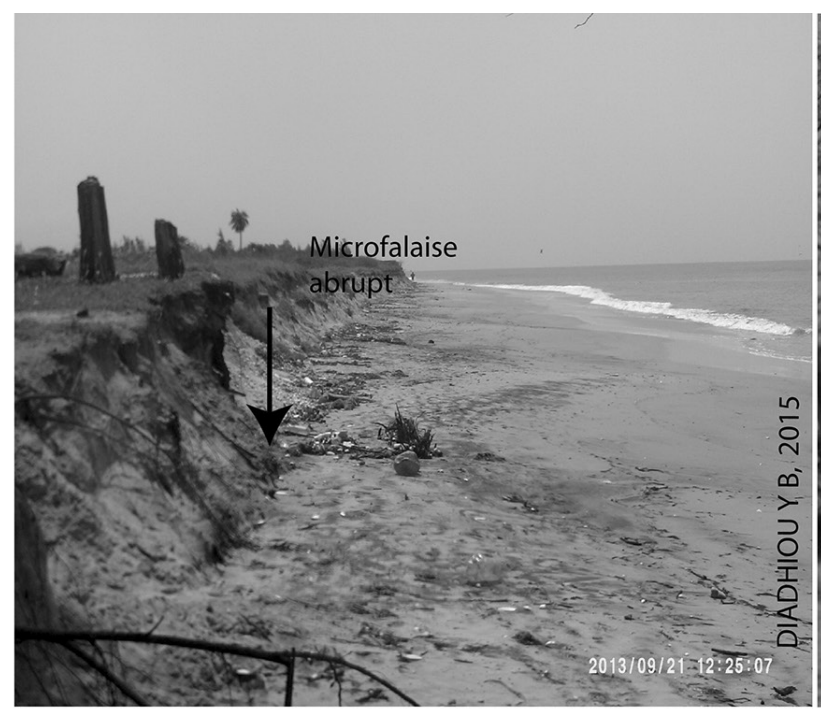

A : Morphologie de la plage vers le nord de Palmarin-Nguéthie Vue en direction sud, 21 septemre 2013

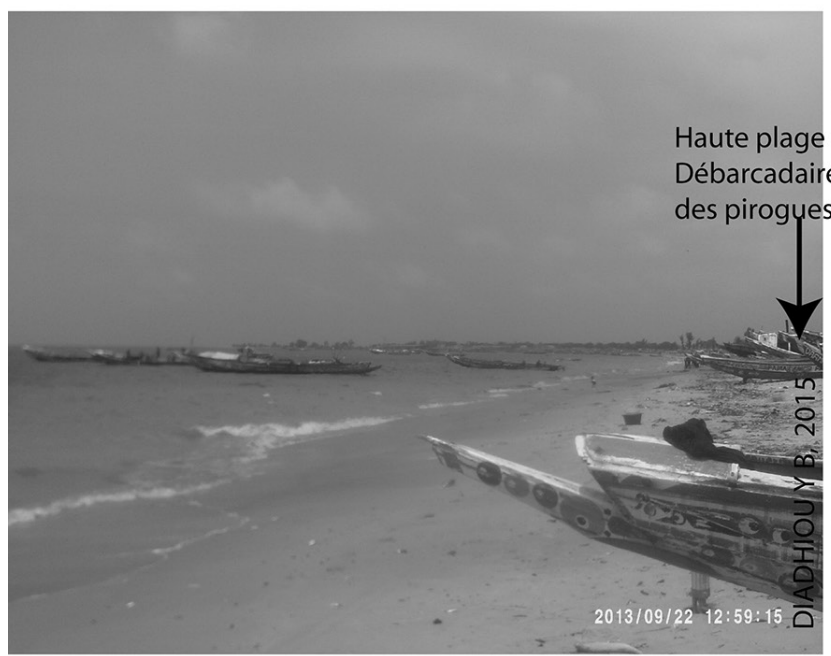

C: Morphologie de la plage vers le quai de pêche Vue en direction nord, 22 septemre 2013

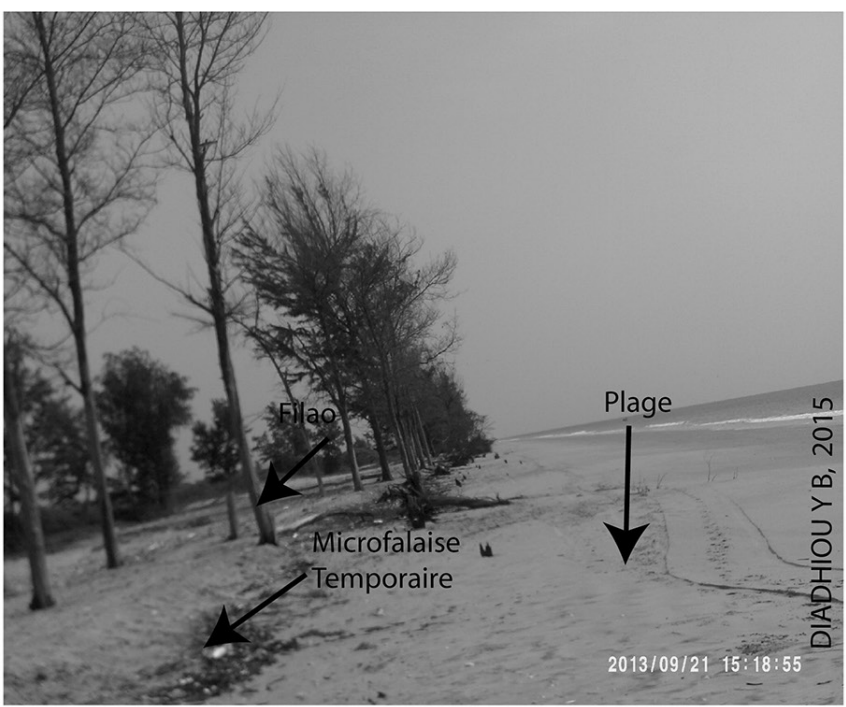

B : Morphologie de la plage vers le Sud de Palmarin-Nguéthie Vue en direction sud, 21 septemre 2013

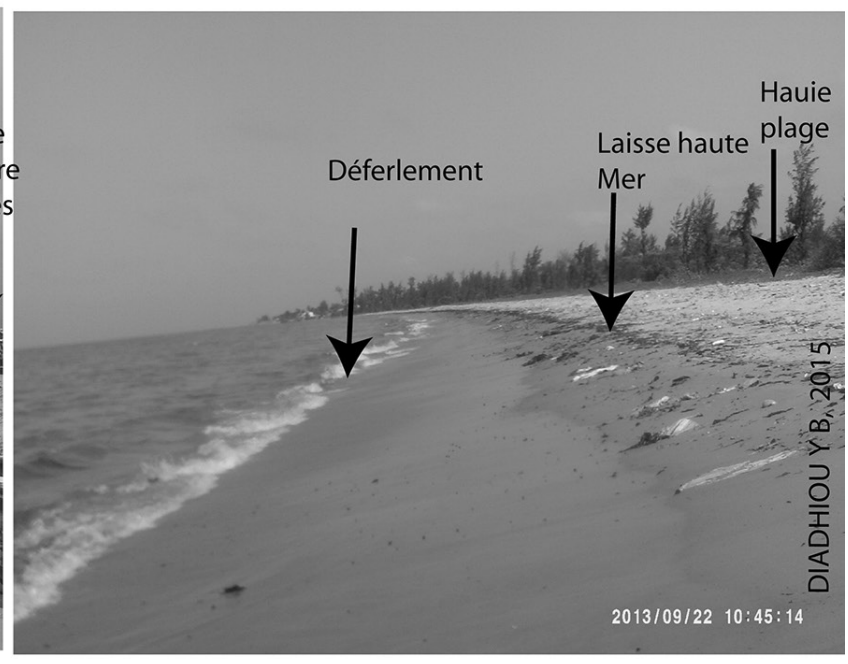

D : Morphologie de la plage sur l'extrémité distale Vue en direction nord, 22 septemre 2013

Figure 7 : Morphologie des plages des flèches de Joal et de Palmarin-Djiffère

Morphology of the beaches of Joal and Palmarin-Diffère spits, photos taken september 21 and 22, 2013

la direction des vents, permet de voir que les déficits les plus importants interviennent pendant l'été, période d'intervention des houles de SW.

À Djiffère, le secteur de Ngallou en érosion est à mettre en rapport avec la morphologie de la côte sur cette partie du littoral qui présente une microfalaise vive permanente. L'hydrodynamique marine en hiver (décembre, janvier, février) et en été (juin, juillet, août) où les vagues (jets de rive) s'abattent sur la côte poussées par les vents de secteur nord-est et d'Ouest (figure 9), amplifient le problème. Les accumulations notées en mars et en septembre n'arrivent pas à contrebalancer les érosions. L'instabilité de la côte de Palmarin-Djiffère, en particulier dans sa partie nord, est instaurée par la brèche. La gradation de l'érosion plus au nord influe sur l'évolution totale de la côte surtout pendant la période des houles sud-ouest obliques à la côte en été ou hivernage (Sall, 1982). 


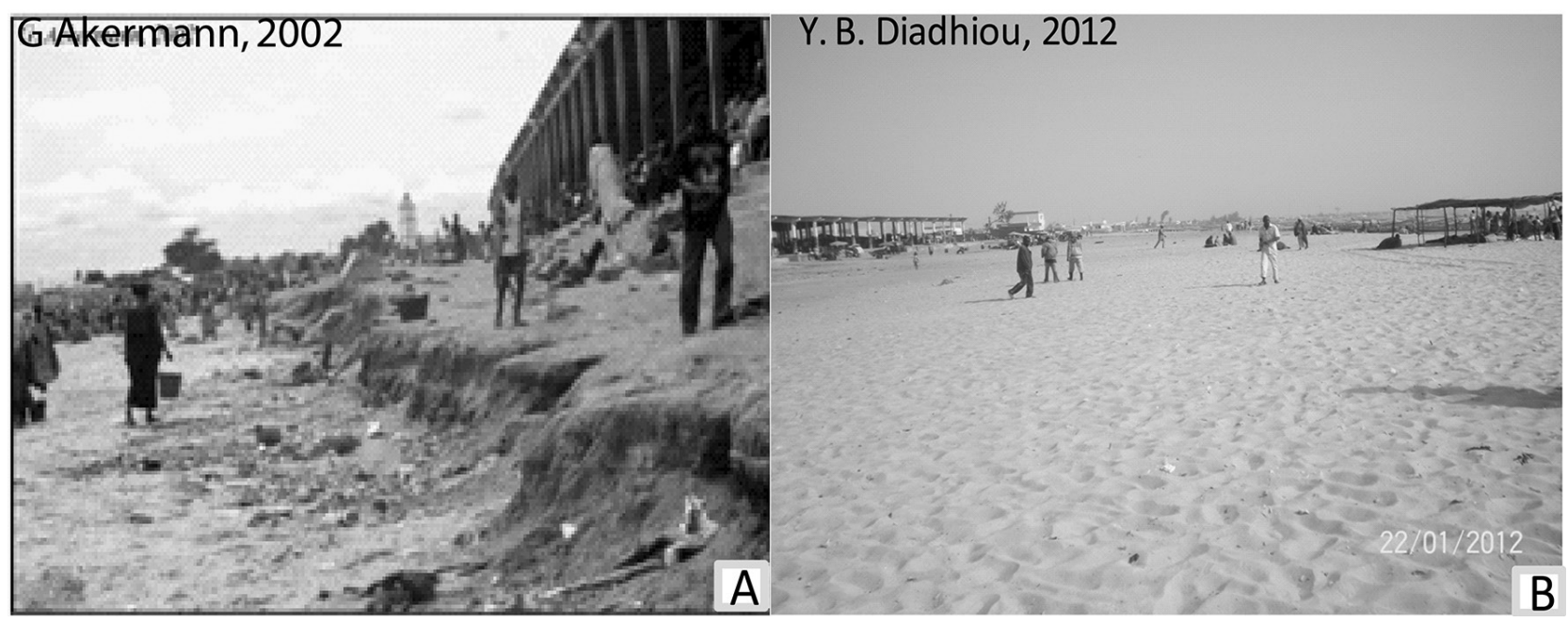

Figure 8 : État de la plage du quai de pêche de Joal entre 2002 et 2012

A : érosion en face du quai de pêche, prise de vue en direction nord en 2002 (d'après Ackermann, 2006); B : Accumulation de la plage du quai de pêche, prise de vue direction sud, 22 janvier 2012 (mission de terrain).

State of the coast between 2002 and 2012 on the beach of the fishing port of Joal

A : erosion in front of the fishing port in 2002 (Ackermann, 2006); B : Accumulation of the beach of the fishing port in january $22,2012$.

\section{Conclusion}

Les études portant sur l'évolution du trait de côte sur les flèches littorales du Sénégal et en particulier celles de Joal et de Palmarin-Djiffère posent plusieurs problèmes en termes d'évaluation des sources d'erreur. Cette étude n'y fait pas exception mais les résultats obtenus sont comparables car la méthode adoptée (la ligne limite des pleines mers ou ligne d'humectation des sables) est la plus utilisée. Les échelles de temps considérées relativement moyennes ou courtes peuvent donner des évolutions du trait de côte plus ou moins importantes. Malgré tout, la dynamique érosive observée, comparée aux données antérieures (Niang-Diop, 1995; Diaw, 1997; Thomas et Diaw, 1997; Diara, 1999; Turmine, 2000 et 2001 ; Sy, 2007 ; Dièye et al., 2013; Diagne, 2015 etc.) s'est confirmée sur la flèche de Palmarin-Djiffère ainsi que l'alternance entre érosion et accumulation des secteurs proximal, médian et extrémité distale à Joal. Cette dynamique fait que la flèche de Joal est assez stable comparée à celle de Djiffère. Cette évolution est en rapport avec l'action des houles, la dérive littorale, la morphologie de la côte, les actions anthropiques et les effets du changement climatique. Elles engendrent soit des pertes soit des gains sédimentaires ou bien facilitent l'action des agents hydrodynamiques et climatiques sur le matériel meuble. Il est certain que la brèche de Sangomar a une part dans la dynamique observée au nord de Djiffère. Le suivi des échanges sédimentaires « cross-shore » sur des profils de plage a permis de saisir l'importance des variations sédimentaires saisonnières le long des segments de plages mesurés : érosion de janvier à février et de juillet à août; et reconstitution partielle en mars et en septembre particulière à Palmarin-Djiffère. Un suivi mensuel quinquennal des échanges sédimentaires et des paramètres hydrodynamiques permettrait de mieux mesurer les interactions de plusieurs agents sur la dynamique côtière de ces flèches.

\section{Remerciements}

Nos remerciements au ministère de l'Enseignement supérieur et de la Recherche du Sénégal qui a financé les travaux par le biais du fond d'impulsion de la recherche scientifique et technique (First). Merci aux relecteurs (M. Alain Hénaff, M. Mohamed Chaibi et M. Grégoire Maillet), à l'équipe de recherche dirigée par Professeur Isabelle Niang et à l'équipe du journal Norois en charge de suivre l'article. 


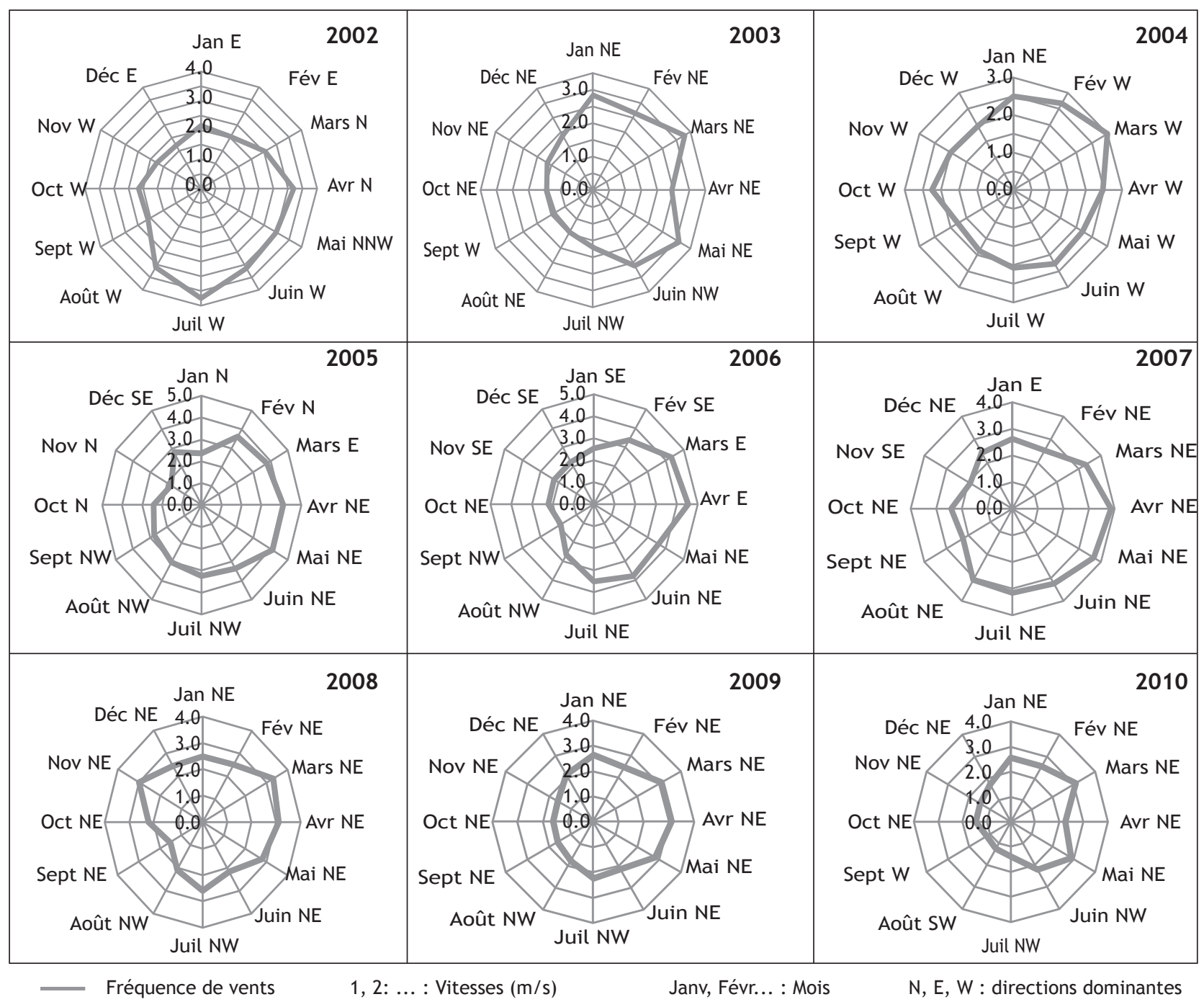

Figure 9 : Direction et vitesse moyenne mensuelle annuelle au sol en m/s des vents à Mbour de 2002 à 2010 (Agence Nationale de l'Aviation Civile et de la Météorologie du Sénégal)

Direction and average annual monthly speed in $\mathrm{m}^{-1} \mathrm{~s}^{-1}$ of the wind in Mbour between 2002 to 2010 (National Agency of Civil aviation and of the Meteorology of Senegal)

\section{Bibliographie}

Ackermann G., Alexandre F., Andrieu J., Mering C., Ollivier C., 2006. Dynamique des paysages et perspectives de développement durable sur la Petite Côte et dans le delta du Sine - Saloum (Sénégal), VertigO, n 2, Vol. 7. DOI : 10.4000/vertigo.2206, [http://vertigo.revues.org/2206].

BÂ K., 2013. Apport de la télédétection et des Sig dans l'étude de l'évolution de la langue de barbarie et de l'estuaire du fleuve Sénégal, thèse de doctorat en géoscience, Université Cheikh Anta Diop, 223 p.

Bird E. C. F., 1985. Coastline changes. A global review, Chichester, A Wiley - Inter-science publication, 219 p.

Bouabdallah M., Larue J.-P., 2009. Évolution du littoral de la baie de Saida : Dynamique naturelle et impacts des aména- gements (Maroc oriental), Physio-Géo Géographie Physique et Environnement, Vol. 3, p. 113-130.

Cesaraccio M., Thomas Y.-F., Diaw A.T., Ouegnimaoua L., 2004. Impact des activités humaines sur la dynamique littorale : prélèvement de sable sur le site de Pointe Sarène, Sénégal, Géomorphologie : relief, processus, environnement, $\mathrm{n}^{\circ}$ 1, vol. 10, p. 55-63.

Cicin-Sain B., Knecht R R.W., 1998. Integrated Coastal and Ocean Management : concepts and practices, Island Press, Washington D.C., $517 \mathrm{p}$

DEEC, 2010. Évolution du trait de côte du littoral de Palmarin; Rapport d'étude de cas au Sénégal, Projet d'Adaptation au changement climatique et côtier (ACCC), Programme Régional de Lutte Contre l'érosion côtière en Afrique de l'Ouest, 77 p. (non publié). 
Diagne B., 2015. Dynamiques climatique et sédimentaire: impacts sur l'évolution des littoraux sableux des localités de Palmarin à Diffère, de Dionewar et de Sangomar (Sénégal), thèse unique de Doctorat en génie littoral, Université Cheikh Anta Diop de Dakar, 278 p.

Diara M., 1999. Formation et Évolution fini-Holocènes et dynamique actuelle du Delta du Saloum-Gambie (Sénégal-Afrique de l'Ouest) - Géomorphologie, Stratigraphie, Sédimentologie et dynamique sédimentaire, thèse de Doctorat en océanographie-géologie, Université de Perpignan, 161 p.

Diaw A. T., 1997. Évolution des milieux littoraux du Sénégal géomorphologie et télédétection. Thèse de doctorat en géographie, Université de Paris I, Panthéon-Sorbonne, 270 p.

Dièye E.B., Diaw A T., Sané T., Ndour N., 2013. Dynamique de la mangrove de l'estuaire du Saloum (Sénégal) entre 1972 et 2010, Cybergeo : European Journal of Geography, Environnement, Nature, Paysage, article 629. DOI : 10.4000/cybergeo.25671. URL : [https://cybergeo.revues. org/25671].

Dolan R., Fenster M.S., Holme S.J., 1991. Temporal analysis of shoreline recession and accretion, Journal of Coastal Research, $\mathrm{n}^{\circ}$ 3, vol. 1, p. 723-744.

Durand P., Heurtefeux H., 2006. Impact de l'élévation du niveau marin sur l'évolution future d'un cordon littoral lagunaire : une méthode d'évaluation. Exemple des étangs de Vic et de Pierre Blanche (littoral méditerranéen, France). Zeitschrift für Geomorphologie N.F., nº 50, vol. 2, p. $221-244$.

Faye I.B.N., 2010. Dynamique du trait de côte sur les littoraux sableux de la Mauritanie à la Guinée-Bissau (Afrique de l'Ouest): Approches régionale et locale par photo-interprétation, traitement d'images et analyse de cartes anciennes, thèse de doctorat en Géographie, Université de Bretagne Occidentale, France, Vol. 1, 321 p

Faye I.B.N., Henaff A., Gourmelon F., Diaw A.T., 2008. Évolution du trait de côte à Nouakchott (Mauritanie) de 1954 à 2005 par photo-interprétation, Norois, n 208, p. 11-27. DOI : 10.4000/norois. 2146.

Minot A., 1934. Étude d'estuaires : Saloum, Paris, BCEOM, $60 \mathrm{p}$.

Nardari B., 1993. Analyse de la houle sur les côtes du Sénégal, application à la Pointe de Sangomar, Rapport de stage UTIS ISRA/CRODT, Université de Toulon et du Var, 31 p.

Ngami-Ntsiba-Andzou P., 2006. Évolution de la lagune de Mbodiène (Petite Côte, Sénégal). Approche par analyse morpho-sédimentaire et Télédétection, thèse de Doctorat de
Troisième cycle en géosciences, Université Cheikh Anta DIOP de Dakar, 214 p.

Niang-Diop I., 1995. L'érosion côtière sur la Petite Côte à partir de l'exemple de Rufisque. Passé - Présent - Futur, thèse de doctorant en géologie, ORSTOM éd., Université d'Angers, tome 1, $318 \mathrm{p}$.

Paskoff R., 1998. La crise des plages : pénurie de sédiments, Mappemonde, 52, p. 11-15.

Pnue, 1989. Méthodologie d'inventaire et de contrôle de l'érosion côtière dans la région de l'Afrique de l'ouest et du centre, Rapport régional, $107 \mathrm{p}$.

SAll M.M., 1982. Dynamique et morphogenèse actuelles au Sénégal occidental, thèse de Doctorat d'État en géographie, Université L. Pasteur, Strasbourg, tome 1, 604 p.

SuANEz S., 2009. La question du bilan sédimentaire des côtes d'accumulation - Rôle des forçages naturels et anthropiques dans les processus morphodynamiques analysés à partir de quelques exemples pris en Méditerranée et en Bretagne, thèse d'Habilitation à Diriger des Recherches, Université de Caen - Basse Normandie, Volume 1, 218 p.

Sy B.A., Bilbao I.A., Sy A.A., Perez I.S., Valido S.R., 2013. Résultats du suivi 2010-2012 de l'évolution de la brèche ouverte sur la Langue de Barbarie au Sénégal et de ses conséquences, Physio-Géo, Volume 7. DOI : 10.4000/physio-geo.3569. URL : [https://physio-geo.revues.org/3569].

Sy B.A., Sy A.A., 2010. Dynamique actuelle du cordon littoral de la Grande Côte sénégalaise de Saint Louis à Niayam et ses conséquences, Revue de Géographie de Saint Louis (RGSL), no 8, p. 197-214.

Sy C., 2007. Impact du quai de pêche sur l'évolution du littoral de Joal, thèse de $3^{\text {e }}$ cycle en Géologie, Université Cheikh Anta Diop de Dakar, 130 p.

Thiam M.D., 1986. Géomorphologie, Evolution et Sédimentologie des Terrains salés du Sine Saloum (Sénégal), thèse $3^{\text {e }}$ cycle en Géographie, Université Paris I, 186 p.

Thomas Y.-F., Diaw A. T., 1997. Suivi (1984-1993) de la rupture de la flèche de Sangomar, estuaire du Saloum, Sénégal. Photo-Interprétation, no 3-4, p 199-204.

Turmine V., 2001. Dynamique littorale entre Mbour et Joal (Petite Côte-Sénégal), mémoire de Maîtrise en Géographie, Université Paris VII - Denis-Diderot, 255 p.

Turmine V., 2000. Les variations spatio-temporelles des marais à mangrove et de leur hydrodynamisme en Afrique de l'Ouest entre la Petite Côte et la Guinée (Géomorphologie et Télédétection), DEA en géographie, Université Paris VII-Denis Diderot, 123 p. 


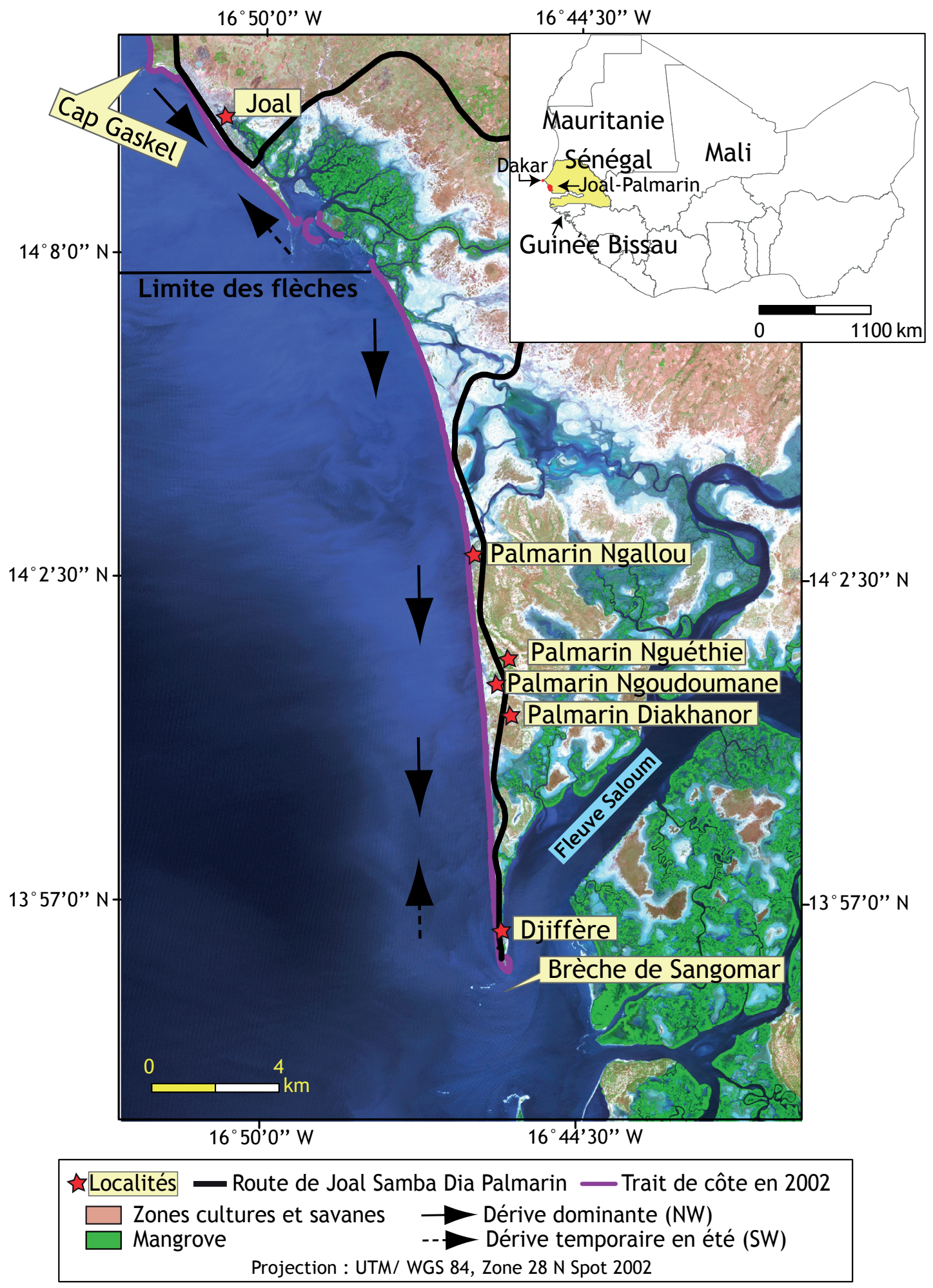

Figure 1 : Environnement côtier sur la flèche de Joal et de Djiffère Coastal environment on the Joal and Djiffère spits 


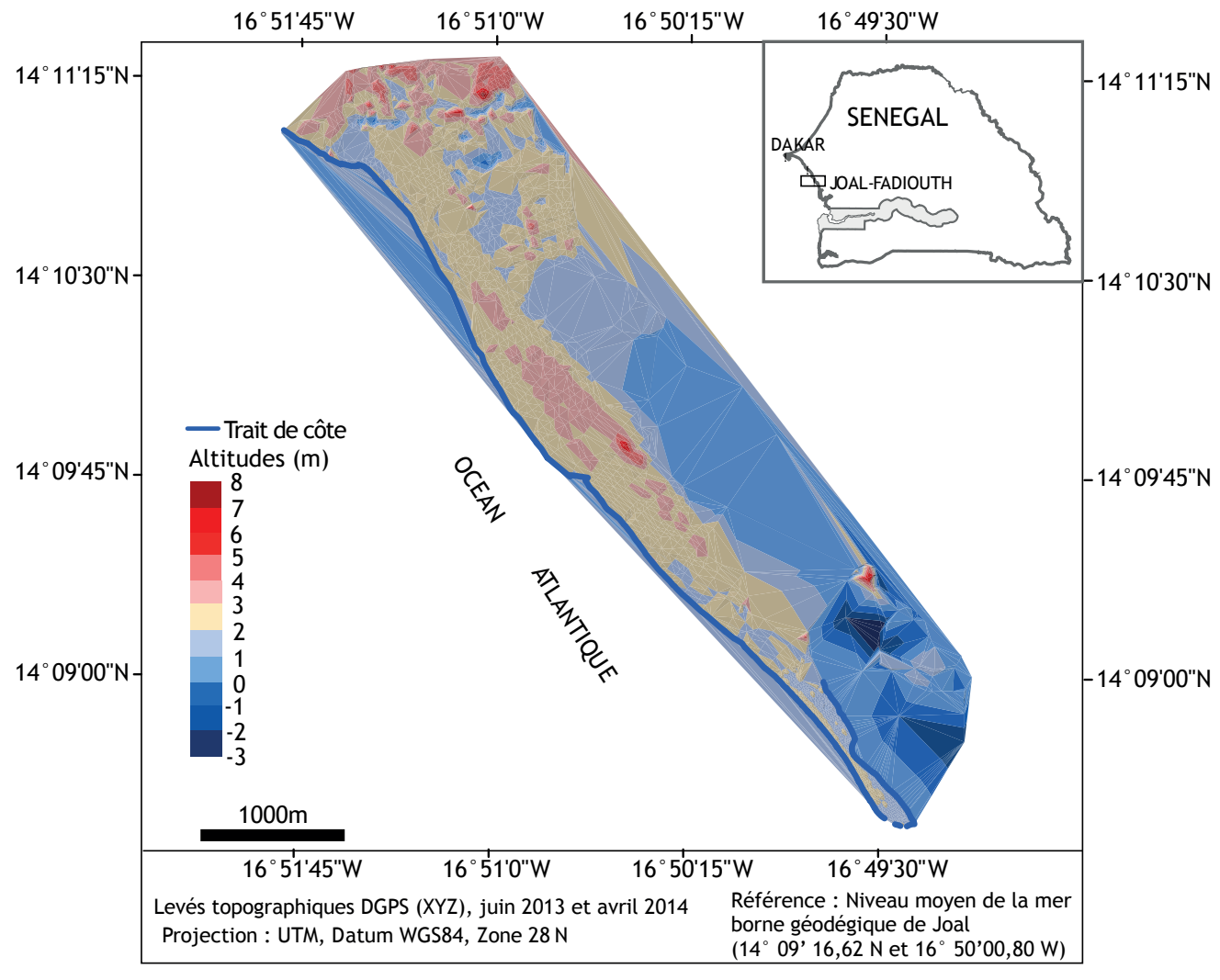

Figure 2 : Altitudes sur la flèche de Joal the Joal sandspit Altitudes

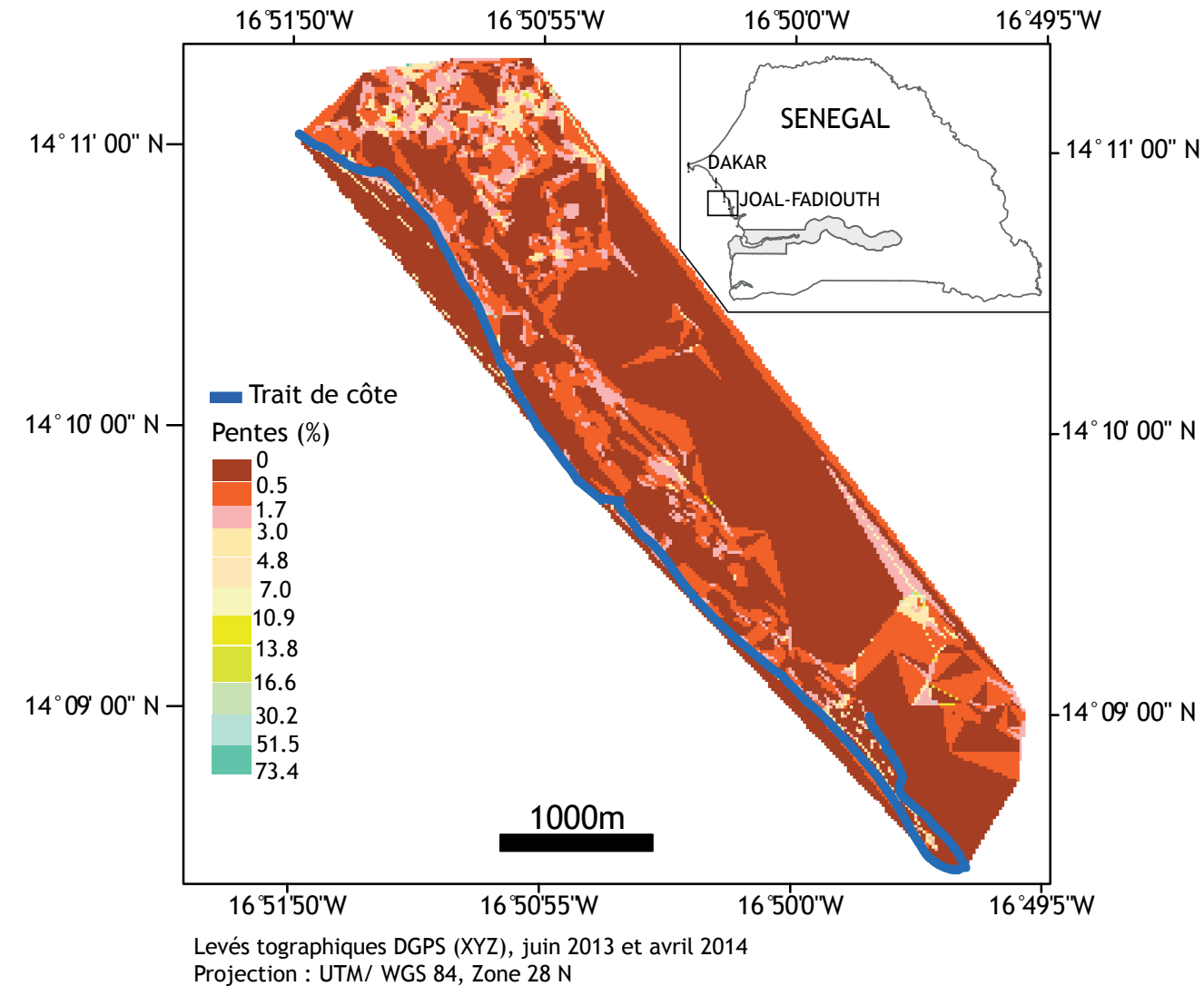




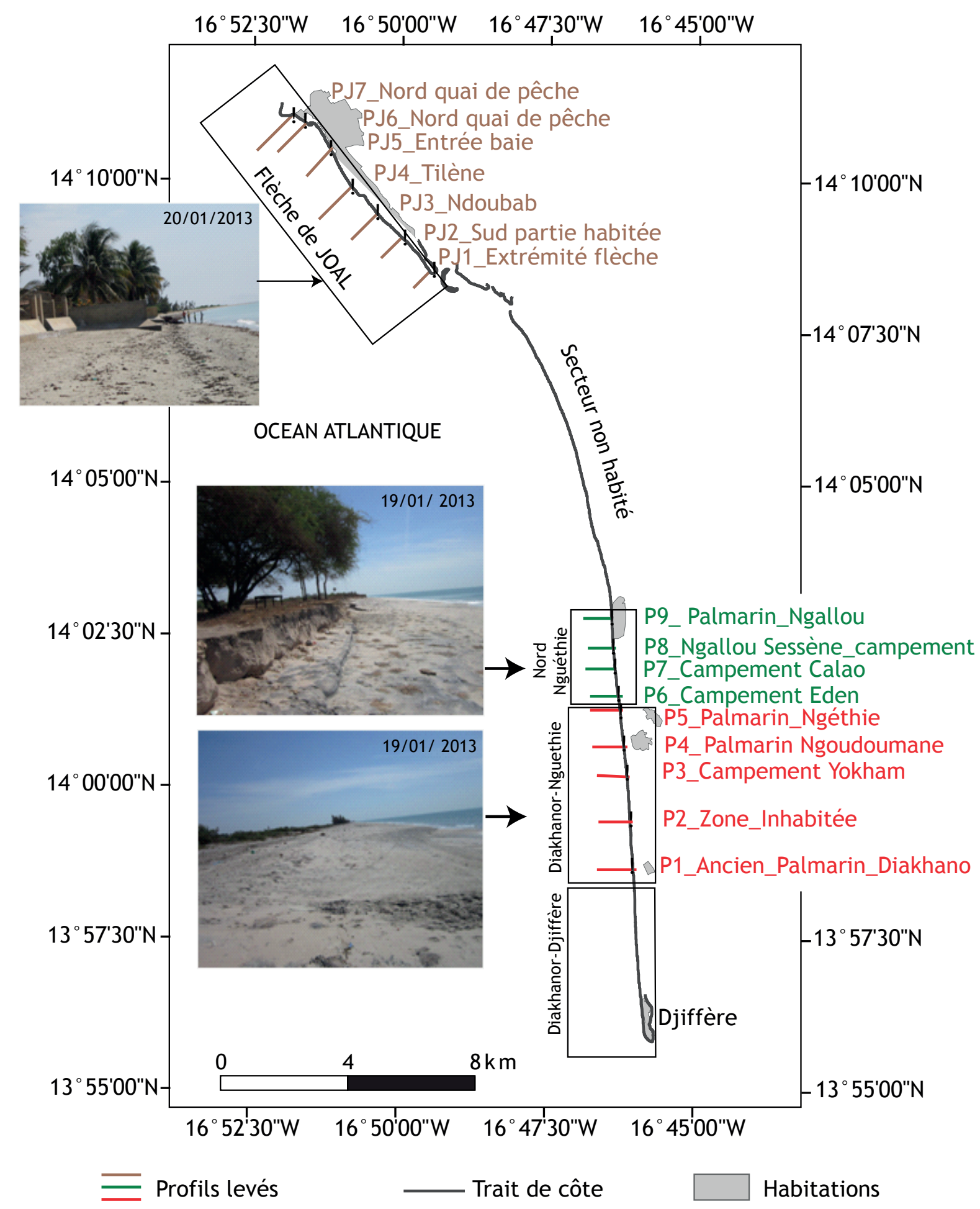

Projection : UTM, WGS 84, Zone $28 \mathrm{~N} \quad$ Carte établie à partir de Spot 2002, Levés GPS

Prise de vue des photos en direction vers le sud

Figure 4 : Localisation des profils de plage sur les flèches de Joal et de Palmarin-Djiffère Localization of beach profiles on the Joal and Palmarin-Djiffère's spits 


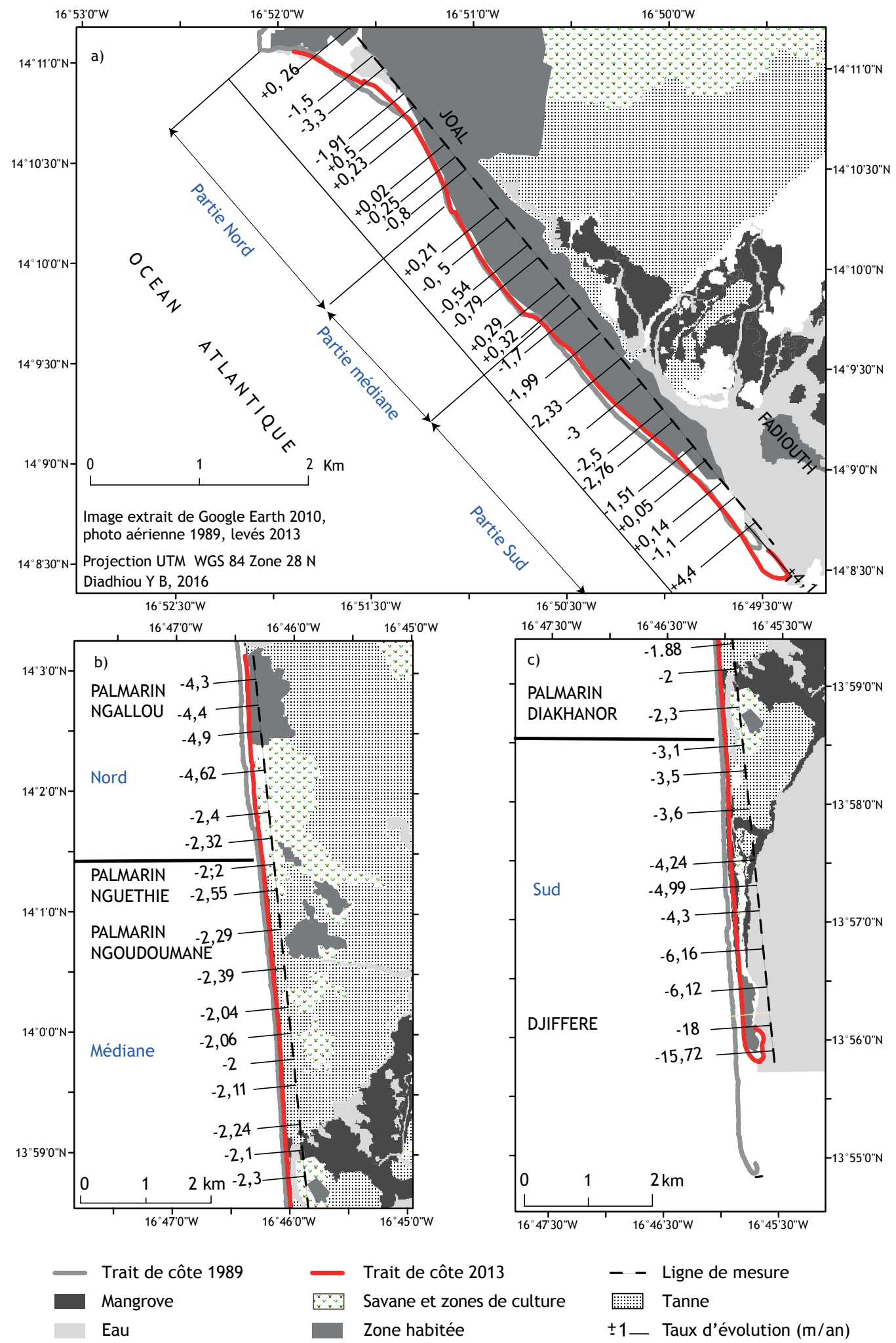

Figure 6 : Taux de recul du trait de côte sur Joal et Palmarin-Djiffère entre 1989 et 2013 (6a : sur la flèche de Joal; 6b au nord de Nguéthie; 6c au sud de Ngoudoumane)

Shoreline retreat rates on Joal and Palmarin-Djiffère between 1989 and 2013 (6a: on the Joal spit; 6b: at the north of Nguethie; 6c: at the south of Ngoudoumane) 